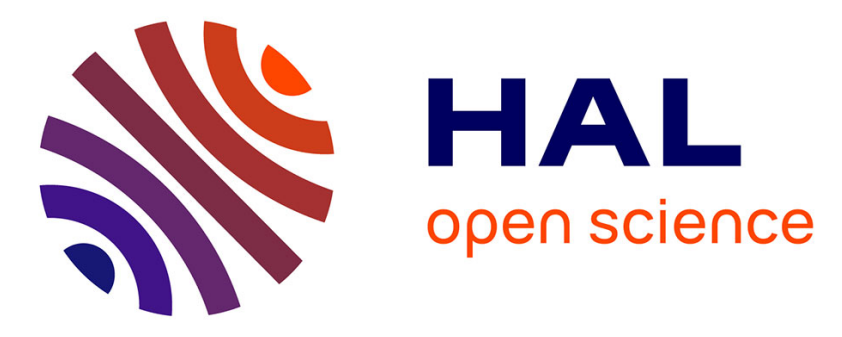

\title{
Relaxation of long-lived modes in NMR of deuterated methyl groups
}

Konstantin L. Ivanov, Thomas Kress, Mathieu Baudin, David Guarin, Daniel Abergel, Geoffrey Bodenhausen, Dennis Kurzbach

\section{- To cite this version:}

Konstantin L. Ivanov, Thomas Kress, Mathieu Baudin, David Guarin, Daniel Abergel, et al.. Relaxation of long-lived modes in NMR of deuterated methyl groups. Journal of Chemical Physics, 2018, 149 (5), pp.054202. 10.1063/1.5031177 . hal-02017760

\section{HAL Id: hal-02017760 \\ https://hal.sorbonne-universite.fr/hal-02017760}

Submitted on 13 Feb 2019

HAL is a multi-disciplinary open access archive for the deposit and dissemination of scientific research documents, whether they are published or not. The documents may come from teaching and research institutions in France or abroad, or from public or private research centers.
L'archive ouverte pluridisciplinaire HAL, est destinée au dépôt et à la diffusion de documents scientifiques de niveau recherche, publiés ou non, émanant des établissements d'enseignement et de recherche français ou étrangers, des laboratoires publics ou privés. 


\title{
Relaxation of long-lived modes in NMR of deuterated methyl groups
}

\author{
Konstantin L. Ivanov, ${ }^{1,2, a)}$ Thomas Kress, ${ }^{3}$ Mathieu Baudin, ${ }^{3}$ David Guarin, ${ }^{3}$ \\ Daniel Abergel, ${ }^{3}$ Geoffrey Bodenhausen, ${ }^{3}$ and Dennis Kurzbach ${ }^{3, a)}$ \\ ${ }^{1}$ International Tomography Center, Siberian Branch, Russian Academy of Science (SB RAS), Institutskaya 3a, \\ Novosibirsk 630090, Russia \\ ${ }^{2}$ Novosibirsk State University, Pirogova 2, Novosibirsk 630090, Russia \\ ${ }^{3}$ Laboratoire des biomolécules, LBM, Département de chimie, École normale supérieure, PSL University, \\ Sorbonne Université, CNRS, 75005 Paris, France
}

\begin{abstract}
Long-lived imbalances of spin state populations can circumvent fast quadrupolar relaxation by reducing effective longitudinal relaxation rates by about an order of magnitude. This opens new avenues for the study of dynamic processes in deuterated molecules. Here we present an analysis of the relaxation properties of deuterated methyl groups $\mathrm{CD}_{3}$. The number of coupled equations that describe cross-relaxation between the 27 symmetry-adapted spin states of a $\mathrm{D}_{3}$ system can be reduced to only 2 non-trivial "lumped modes" by (i) taking the sums of the populations of all states that equilibrate rapidly within each irreducible representation of the symmetry group, and (ii) by combining populations that have similar relaxation rates although they belong to different irreducible representations. The quadrupolar relaxation rates of the spin state imbalances in $\mathrm{CD}_{3}$ groups are determined not by the correlation time of overall tumbling of the molecule, but by the frequency of jumps of methyl groups about their three-fold symmetry axis. We access these states via dissolution dynamic nuclear polarization (D-DNP), a method that allows one to populate the desired long-lived states at cryogenic temperatures and their subsequent detection at ambient temperatures after rapid dissolution. Experimental examples of DMSO- $\mathrm{d}_{6}$ and ethanol- $\mathrm{d}_{6}$ demonstrate that long-lived deuterium spin states are indeed accessible and that their lifetimes can be determined. Our analysis of the system via "lumped" modes allows us to visualize different possible spin-state populations of symmetry A, B, or E. Thus, we identify a long-lived spin state involving all three deuterons in a $\mathrm{CD}_{3}$ group as an $\mathrm{A} / \mathrm{E}$ imbalance that can be populated through DNP at low temperatures.
\end{abstract}

\section{NOMENCLATURE}

DNP = dynamic nuclear polarization

D-DNP = dissolution dynamic nuclear polarization

NMR = nuclear magnetic resonance

LLS = long-lived state

SSI $=$ spin state imbalance

\section{INTRODUCTION}

In nuclear magnetic resonance (NMR), the combination of long-lived spin states (LLSs) $)^{9,10,17,20,21}$ with hyperpolarization methods based on dissolution dynamic nuclear polarization $(\mathrm{D}-\mathrm{DNP})^{3}$ has the potential to preserve nuclear hyperpolarization over periods of time much longer than spin-lattice relaxation times, which opens new avenues for the study of slow chemical exchange $e^{1,8,24}$ and slow transport phenomena. ${ }^{2,22,25}$ An illustrative example of an LLS is the singlet state of a pair of spins with $I=1 / 2$, such as a pair of protons in $\mathrm{H}_{2}$ molecules or $-\mathrm{CH}_{2}-$ fragments. Since the intra-pair dipolar coupling is symmetric with respect to spin exchange, it cannot induce

a) Authors to whom correspondence should be addressed: Ivanov@ tomo.nsc.ru and Kurzbach@ens.fr transitions between the anti-symmetric singlet state and the symmetric triplet states. Such forbidden transitions can only be induced by extra-pair dipole-dipole interactions, paramagnetic effects, anisotropic chemical shifts, or spin rotation effects. However, as dipolar relaxation by intra-pair dipolar couplings is often dominant, the lifetime of imbalances between the populations of singlet and triplet states can be much longer than spin-lattice relaxation times, provided the intramolecular dipolar coupling is efficiently suppressed. For quantitative calculations of the lifetimes of population imbalances between manifolds of different symmetry, cross correlations of the fluctuations of distinct dipole-dipole interactions must be considered, but auto-correlations of dipolar interactions suffice for a qualitative understanding of the flow of populations between manifolds of different symmetry. $\operatorname{In}{ }^{13} \mathrm{C}^{1} \mathrm{H}_{3}$ groups, cross-relaxation between spin states that belong to different irreducible representations $\mathrm{A}, \mathrm{B}, \mathrm{E} 1$, and $\mathrm{E} 2$ of the $\mathrm{C}_{3 \mathrm{v}}$ point group can only be explained by correlated fluctuations of the ${ }^{1} \mathrm{H}-{ }^{1} \mathrm{H}$ and ${ }^{1} \mathrm{H}-{ }^{13} \mathrm{C}$ dipole-dipole interactions. ${ }^{12}$

LLSs have been studied ${ }^{12,13,18}$ in many systems comprising two or more protons or heteronuclei such as ${ }^{13} \mathrm{C},{ }^{19} \mathrm{~F}$, etc. Molecules containing at least two magnetically equivalent deuterium nuclei that have spin $S=1$ (in this paper, we shall use the symbol " $\mathrm{D}$ " rather than ${ }^{2} \mathrm{H}$ for simplicity) 
imbalances between populations of spin states of different symmetry can give rise to LLS, in the sense that their lifetime $T_{L L S}(\mathrm{D})$ can be longer than their Zeeman spin-lattice relaxation times $T_{1}(\mathrm{D})$, which are usually short because of rapid quadrupolar relaxation of deuterium nuclei. ${ }^{15,16}$ In the extreme narrowing regime, deuterium spin-lattice relaxation rates $R_{1}(\mathrm{D})=1 / T_{1}(\mathrm{D})$ are proportional to $\omega_{Q}^{2} \tau_{C}$ where $\omega_{Q}$ is the quadrupolar coupling and $\tau_{C}$ is the correlation time of molecular tumbling.

To create LLSs in deuterated ${ }^{13} \mathrm{CD}_{2}$ and ${ }^{13} \mathrm{CD}_{3}$ groups, we employed dissolution dynamic nuclear polarization (D-DNP), ${ }^{15}$ which allows one to generate imbalances between the averaged populations of the A-states and E-states, thus giving rise to long-lived states.

A description of LLS in ${ }^{13} \mathrm{CD}_{2}$ groups has been outlined elsewhere. ${ }^{15}$ The nine energy states of the $\mathrm{D}_{2}$ subsystem can be separated into six sextet states that are symmetric with respect to the permutation of the two deuterium nuclei, and three triplet states are anti-symmetric upon permutation. The LLS has been identified as a "sextet-triplet imbalance" (STI). To evaluate the lifetime $T_{S T I}$, a $18 \times 18$ relaxation matrix can be constructed containing relaxation rates that have been determined using a model assuming that (i) relaxation was driven by quadrupolar relaxation, the two quadrupolar tensors being assumed to be axially symmetric and aligned along the ${ }^{13} \mathrm{C}-\mathrm{D}$ bonds, and (ii) molecular motion was assumed to be described by anisotropic rotational diffusion so that the two quadrupolar tensors were in effect averaged about the two-fold axis. $^{15}$

The analysis of LLSs in ${ }^{12} \mathrm{CD}_{3}$ groups is more complex since the system comprises 27 states. (Extensions to ${ }^{13} \mathrm{CD}_{3}$ groups that comprise 54 states will not be considered here. If we consider a deuterated methyl group containing a ${ }^{13} \mathrm{C}$ spin, the stepwise reduction can be conducted in a similar manner, provided one neglects multispin order involving the carbon nucleus.)

The relaxation matrix can be constructed ${ }^{15}$ in the basis of symmetry-adapted spin states as originally devised by Bernatowicz et al. ${ }^{6}$ The symmetry-adapted states are discussed in the Appendix. The origin of LLSs can be ascribed to generalized spin-state imbalances (SSIs), which can be identified qualitatively as population imbalances that persist due to the inefficient flow of populations between spin states belonging to different irreducible representations. However, the actual long-lived population modes were not identified, and their lifetimes (i.e., the inverse of their relaxation times) have not yet been determined and remain an open question. One should note that the case of ${ }^{13} \mathrm{CD}_{3}$ groups is significantly more complex compared to the ${ }^{13} \mathrm{CD}_{2}$ case, not only because of the much larger relaxation matrix, but also because the symmetry-adapted states do not merely belong to two, but to four distinct symmetry manifolds (A, B, E1, and E2). Several population imbalances that can potentially lead to LLSs need therefore to be considered, since there are 6 pairs of manifolds.

In this work, we present an approach that allows one to reduce the dimensionality of the relaxation matrix of a $\mathrm{D}_{3}$ group from $27 \times 27$ to $3 \times 3$. This enables the determination of long-lived modes of "lumped populations" as well as their relaxation rates and lifetimes. These modes can be monitored indirectly by inspection of the scalar-coupled multiplet in the ${ }^{13} \mathrm{C}$ NMR spectrum of a ${ }^{13} \mathrm{CD}_{3}$ group. This indirect observation enables the quantitative interpretation of experimental results and provides an understanding of the key factors governing the relaxation behavior of LLSs in deuterated methyl groups.

An extension to protonated methyl groups is straightforward, although one has to take into account 3 homoand 3 heteronuclear dipole-dipole couplings and 4 chemical shift anisotropy (CSA) tensors and a variety of cross correlations between their fluctuations, rather than merely 3 dominant quadrupolar interactions. Yet, due to the much smaller dimension of the Zeeman space $(8 \times 8$ instead of $27 \times 27)$, the theoretical treatment can proceed without the reduction of the dimension of Liouville space demonstrated here.

\section{THEORY}

The approach developed here has been described in a previous publication ${ }^{15}$ where the $27 \times 27$ dimensional relaxation matrix $\mathbf{R}$ had been introduced. Only relaxation mechanisms due to quadrupolar couplings have been considered, all other mechanisms being neglected. Two molecular motions are considered in this model: (i) isotropic tumbling of the molecule with a correlation time $\tau_{C}$ and (ii) rotation of the methyl group around the $\mathrm{C}-\mathrm{C}$ bond with a correlation time $\tau_{R}$. The model has been implemented in Mathematica using the SpinDynamica package, adapted from the work by Levitt and co-workers for protons. ${ }^{4}$ The $\mathbf{R}$-matrix has been calculated in the Redfield approximation in the extreme narrowing limit. Previous work had revealed two types of long-lived states in ${ }^{13} \mathrm{CD}_{2}$ and ${ }^{13} \mathrm{CD}_{3}$ groups, (i) one in-phase with the attached carbon and (ii) another in antiphase with respect to the carbon nucleus. They give rise to (i) deviations of the relative intensities of the carbon-13 septet from the ideal hightemperature 1:3:6:7:6:3:1 distribution, in such a manner that the central line has varying intensity with respect to the outer lines and (ii) an asymmetry of the septet with respect to its center. The latter effect is associated with ${ }^{13} \mathrm{C}-\mathrm{D}$ multi-spin order terms and is not considered here, since its description requires an extension of the dimension of $\mathbf{R}$ to $54 \times 54$ to include the carbon-13 nucleus. We focus on the LLS associated with population imbalances of the spin states of the 3 deuterons.

Generally, relaxation processes of populations involving $N$ states of the symmetry-adapted basis can be described in Liouville space, where the $N \times N$-dimensional density matrix $\rho$ can be developed to form an $N^{2}$-dimensional columnvector, while the relaxation rates given by the relaxation superoperator $\widehat{\hat{R}}$ can be expressed by an $N^{2} \times N^{2}$-dimensional matrix $\mathbf{R}$. The auto-relaxation rate $R_{i i}$ of the population of the $i$ th state corresponds to the diagonal element of the matrix $\mathbf{R}$, whereas the cross-relaxation rate $R_{j i}$ that describes the flow between populations $p_{i}$ and $p_{j}$ corresponds to the off-diagonal element $R_{j j, i i}$ between two elements $\rho_{i i}$ and $\rho_{j j}$. The secular approximation allows us to neglect all elements that connect populations and spin coherences, apart from zero-quantum 
coherences (ZQCs) between degenerate states. Hence, we can omit all coherences of order $p \geq 1$ and need not consider the entire $N^{2} \times N^{2}$-dimensional matrix $\mathbf{R}$ by taking only the elements that are responsible for the interconversion of the populations and ZQCs. We shall describe the column-vector of state populations $\mathbf{P}$, comprising the elements $\{\mathbf{P}\}_{i}=p_{i}$, and the relaxation matrix $\mathbf{R}$ comprising the following elements:

$$
\mathbf{R}_{i j}=-R_{i i, j j}=-R_{j i} \quad \text { when } \quad i \neq j ; \quad \mathbf{R}_{i i}=\sum_{j \neq i} R_{i j} .
$$

Here we consider the $\mathbf{R}$-matrix as symmetric. The time evolution of the $\mathbf{P}$-vector obeys the following differential equation:

$$
\frac{d \mathbf{P}}{d t}=-\mathbf{R}\left(\mathbf{P}-\mathbf{P}_{t h}\right)
$$

where $\mathbf{P}_{t h}$ stands for the thermal populations of the nuclear spin states.

In $\mathrm{CD}_{3}$ groups, the elements of the $27 \times 27$-dimensional matrix $\mathbf{R}$ are the rates of auto- and cross-relaxation between the populations of the 27 different states of the 3 deuterium spins, using the symmetry-adapted basis defined by Bernatowicz et al. ${ }^{6}$ A graphical representation of this relaxation matrix is depicted in Fig. 1 for the case where $\tau_{C} \gg \tau_{R}$ together with the set of symmetry-adapted manifolds of spin states. For Fig. 1(a), the states have been ordered according to the irreducible representations, leading to a block-diagonal form, showing the lack of cross-relaxation between different symmetry manifolds; this feature allows LLSs to occur. In Fig. 1(a), the colors reflect the cross-relaxation rates (offdiagonal elements shown in white are very small although they are not necessarily zero). One should note that such an appearance of the $\mathbf{R}$-matrix, corresponding to fast relaxation within manifolds of similar symmetry and negligible relaxation between different manifolds, is expected only when there is a hierarchy of motional correlation times, $\tau_{C} \gg \tau_{R}$.
First step in the reduction of the dimension. From Fig. 1, it is obvious that some of the off-diagonal elements of the relaxation matrix are large (represented by dark colors), meaning that the cross-relaxation rates are fast, while some others are small so that cross-relaxation can be neglected. For the individual states within each manifold, we can assume that they rapidly acquire identical populations since all populations within each spin manifold are equilibrated rapidly by crossrelaxation. For example, we can average the populations of the $7+3=10$ states of symmetry A. Likewise, we can average the populations of the $5+3=8$ states of symmetry E1 and of the $5+3=8$ states of symmetry E2. Thus, we can group the state populations by adding populations belonging to the same irreducible representations,

$$
\mathcal{P}_{A}=\sum_{i=1}^{10} p_{i}, \mathcal{P}_{B}=p_{11}, \mathcal{P}_{E 1}=\sum_{i=12}^{19} p_{i}, \mathcal{P}_{E 2}=\sum_{i=20}^{27} p_{i}
$$

This amounts to a reduction of the dimension of Liouville space from $27 \times 27$ to $4 \times 4$. This assumption allows us to sum the corresponding equations in system (2) and to obtain the equations for the populations $\mathcal{P}_{A}, \mathcal{P}_{E 1}$, and $\mathcal{P}_{E 2}$. For example, for the A-states we write

$$
\begin{aligned}
\frac{d \mathcal{P}_{A}}{d t}=\sum_{i=1}^{10} \frac{d p_{i}}{d t}= & -\sum_{i=1}^{10} \sum_{j=1}^{27} \mathbf{R}_{i j} \Delta p_{j} \\
= & -\mathbf{R}_{11}^{\prime} \Delta \mathcal{P}_{A}-\mathbf{R}_{12}^{\prime} \Delta \mathcal{P}_{E 1} \\
& -\mathbf{R}_{13}^{\prime} \Delta \mathcal{P}_{E 2}-\mathbf{R}_{14}^{\prime} \Delta \mathcal{P}_{B} .
\end{aligned}
$$

Here we introduce the elements of the relaxation matrix $R^{\prime}$ in the reduced $4 \times 4$ space. The elements can be calculated as follows:

$$
\begin{array}{ll}
\mathbf{R}_{11}^{\prime}=\frac{1}{10} \sum_{i=1}^{10} \sum_{j=1}^{10} \mathbf{R}_{i j}, & \mathbf{R}_{12}^{\prime}=\sum_{i=1}^{10} \mathbf{R}_{i, 11}, \\
\mathbf{R}_{13}^{\prime}=\frac{1}{8} \sum_{i=1}^{10} \sum_{j=12}^{19} \mathbf{R}_{i j}, & \mathbf{R}_{14}^{\prime}=\frac{1}{8} \sum_{i=1}^{10} \sum_{j=20}^{27} \mathbf{R}_{i j} .
\end{array}
$$

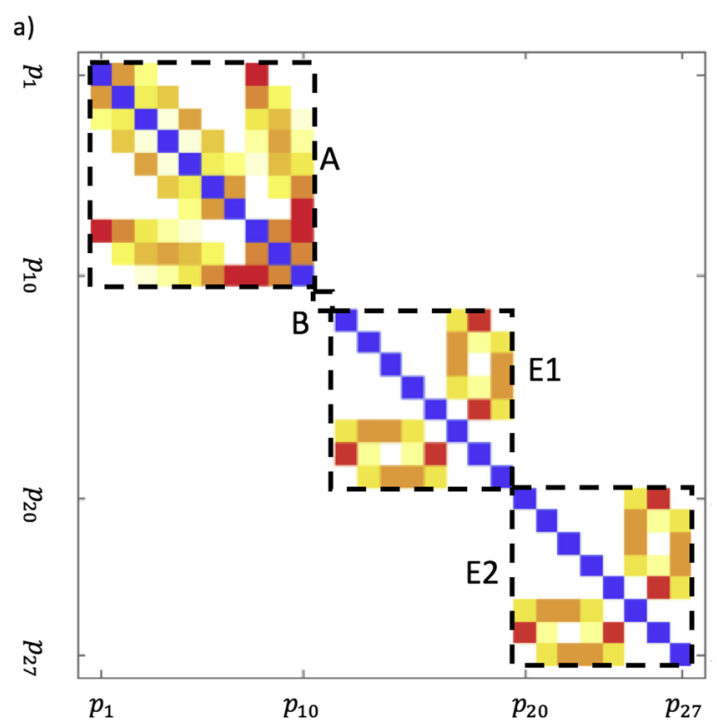

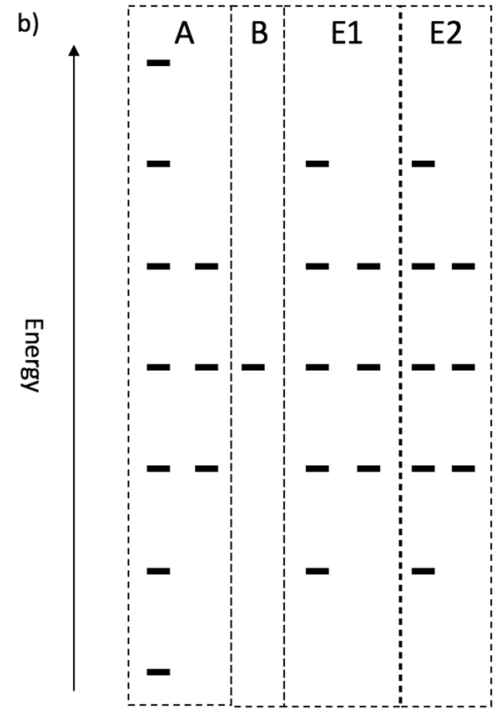

FIG. 1. (a) Pictorial representation of the $27 \times 27$ matrix $\mathbf{R}$ describing relaxation of the 27 populations associated with the three deuterons in a ${ }^{12} \mathrm{CD}_{3}$ group. The inclusion of a coupled ${ }^{13} \mathrm{C}$ nucleus would double the size of the matrix. Blue shades indicate negative auto-relaxation rates, while colors from yellow to red stand for positive cross-relaxation rates. The irreducible representations A, E1, E2, and B are indicated. White off-diagonal elements correspond to small but possibly non-vanishing crossrelaxation rates. The matrix corresponds to the case $\tau_{R} \ll \tau_{C}$. (b) Energy level diagram of the 27 symmetryadapted spin states of the deuterons in a $\mathrm{CD}_{3}$ group; explicit eigenfunctions are given by Bernatowicz et al. ${ }^{6}$ 
All other elements of $\mathbf{R}^{\prime}$ can be evaluated in a similar way. In contrast to $\mathbf{R}$, the $\mathbf{R}^{\prime}$ matrix is no longer symmetric, resulting in unequal populations of the lumped states at thermal equilibrium, as discussed below. By lumping together the populations within the same manifold, we can introduce a new 4-dimensional column-vector of populations $\mathcal{P}$ with the elements $\mathcal{P}_{A}, \mathcal{P}_{E 1}, \mathcal{P}_{E 2}$, and $\mathcal{P}_{B}$ and construct a new differential equation with a $4 \times 4$ relaxation matrix,

$$
\frac{d \mathcal{P}}{d t}=-\mathbf{R}^{\prime}\left(\mathcal{P}-\mathcal{P}_{t h}\right)
$$

The elements of $\mathcal{P}_{t h}$ are the thermal populations of the A, $\mathrm{E} 1, \mathrm{E} 2$, and B states. The new relaxation matrix $\mathbf{R}^{\prime}$ can be diagonalized analytically. Its eigenvalues $\lambda_{k}$ give the effective auto-relaxation rates of the averaged populations. One of the modes, $\lambda_{1}$, is always zero corresponding to the fact that the sum of all four lumped populations must always remain equal to 1 . The corresponding eigenvector $\mathcal{V}_{1}$ is simply a weighted sum of the four averaged populations,

$$
\mathcal{V}_{1}=\frac{1}{\sqrt{229}}\left(\begin{array}{c}
10 \\
1 \\
8 \\
8
\end{array}\right)
$$

The weights of this vector are equal to the number of states in the corresponding batches of averaged states. The remaining three normalized eigenvectors are found to be,

$$
\mathcal{V}_{2}=\frac{1}{2}\left(\begin{array}{c}
1 \\
1 \\
-1 \\
-1
\end{array}\right), \mathcal{V}_{3}=\frac{1}{\sqrt{42}}\left(\begin{array}{c}
5 \\
-1 \\
-4 \\
0
\end{array}\right), \mathcal{V}_{4}=\frac{1}{\sqrt{2}}\left(\begin{array}{c}
0 \\
0 \\
1 \\
-1
\end{array}\right)
$$

At thermal equilibrium, only the $\mathcal{V}_{1}$ mode is populated, since the other three relax to naught due to multiplication by exponents $e^{-\lambda_{k} t}$, which tend to zero for large $t$ since the relaxation rates are positive $\lambda_{k}>0$.

Long-lived modes. The non-vanishing $\lambda_{k}$ rates have been determined analytically by using Mathematica supplemented with a SpinDynamica ${ }^{4}$ routine to calculate the $\mathbf{R}$-matrix, to evaluate the $\mathbf{R}^{\prime}$-matrix, and to determine the eigenvalues. Such a treatment yields the following values of $\lambda_{k}$ :

$$
\begin{aligned}
& \lambda_{2}=\frac{3}{2} \lambda_{3}, \\
& \lambda_{3}=\lambda_{4}=\frac{81}{64} \tau_{C} \tau_{R} \omega_{Q}^{2} \sin ^{2} \theta \frac{17 \tau_{C}+5 \tau_{R}+3\left(5 \tau_{C}+\tau_{R}\right) \cos 2 \theta}{\left(\tau_{C}+\tau_{R}\right)\left(4 \tau_{C}+\tau_{R}\right)},
\end{aligned}
$$

where $\theta=70.5^{\circ}$ is the angle between the three-fold symmetry axis of the methyl group and the CD-bonds so that $\sin \theta=\frac{2 \sqrt{2}}{3}$ and $\cos \theta=\frac{1}{3}$, yielding

$$
\begin{aligned}
& \lambda_{2}=\frac{9 \tau_{C} \tau_{R}\left(2 \tau_{C}+\tau_{R}\right)}{2\left(\tau_{C}+\tau_{R}\right)\left(4 \tau_{C}+\tau_{R}\right)} \omega_{Q}^{2}, \\
& \lambda_{3}=\lambda_{4}=\frac{3 \tau_{C} \tau_{R}\left(2 \tau_{C}+\tau_{R}\right)}{\left(\tau_{C}+\tau_{R}\right)\left(4 \tau_{C}+\tau_{R}\right)} \omega_{Q}^{2}
\end{aligned}
$$

where all eigenvalues have units of $\mathrm{s}^{-1}$. Already at this stage, the analysis reveals the existence of long-lived modes. Indeed, if we assume that $\tau_{R} \ll \tau_{C}$ (a common assumption for methyl group dynamics), the relaxation rates are independent of $\tau_{C}$,

$$
\lambda_{2}=\frac{9}{4} \omega_{Q}^{2} \tau_{R}, \lambda_{3}=\lambda_{4}=\frac{3}{2} \omega_{Q}^{2} \tau_{R}
$$

Since the longitudinal Zeeman relaxation rate is

$$
R_{1}(\mathrm{D})=\tau_{c} \omega_{Q}^{2}\left(9+24 \frac{\tau_{R}}{\tau_{R}+\tau_{C}}+48 \frac{\tau_{R}}{\tau_{R}+4 \tau_{C}}\right),
$$

we obtain the following ratio:

$$
\frac{3 \lambda_{2}}{2 R_{1}}=\frac{\lambda_{3}}{R_{1}}=\frac{\lambda_{4}}{R_{1}}=\frac{3 \tau_{R}\left(\tau_{R}+2 \tau_{C}\right)}{4\left(4 \tau_{C}^{2}+21 \tau_{C} \tau_{R}+9 \tau_{R}^{2}\right)} .
$$

If we assume again that $\tau_{R} \ll \tau_{C}$,

$$
\frac{\lambda_{2}}{R_{1}}=\frac{3 \tau_{R}}{8 \tau_{C}} \ll 1 .
$$

Hence, the specified modes relax much slower than zmagnetization. The reason is that upon fast rotation of a $\mathrm{CD}_{3}$ group, the three quadrupolar interaction tensors are effectively averaged so that the resulting mean quadrupolar interaction is invariant upon rotation. Consequently, crossrelaxation between states belonging to manifolds of different symmetries is suppressed. Since dipolar relaxation is also unable to drive cross-relaxation between such states, one can expect to see long-lived populations modes in $\mathrm{CD}_{3}$ systems. A similar reasoning has already been emphasized in 1979 by Poupko et al. ${ }^{23}$ The authors demonstrated that transitions between states of different irreducible representations vanish when the two spins are equivalent and the corresponding interaction tensors are aligned. In other words, any flow of population between states of different symmetry that involves one or more degenerate spins is forbidden to first order. In the case under study, this means that if a $\mathrm{CD}_{3}$ group rotates so fast that the three deuterons are no longer discernible, one or several LLS due to an SSI can be expected. On the contrary, we do not expect long-lived relaxation modes for $\mathrm{CD}_{3}$ groups featuring high energy barriers and slow rotation around the $C_{3 \mathrm{v}}$ axis (where the assumption $\tau_{R} \ll \tau_{C}$ breaks down) since the three deuterons are no longer equivalent.

Note that the above equations are based on analytical solutions of the entire product operator space, prior to the reduction of the dimensions, so that all coherences are considered in addition to the populations. Note that zero-quantum coherences spanning nearly degenerate energy states can oscillate with relatively low frequencies and may perturb the dynamic separation of different symmetry manifolds, as discussed in some detail in earlier work. ${ }^{5,7,16}$

Second step in the reduction of the dimension. To simplify the problem even further, we note that, first, two modes $\mathcal{V}_{3}$ and $\mathcal{V}_{4}$ have identical relaxation rates $\lambda_{3}=\lambda_{4}$ and, second, the difference in the populations of the E1 and E2 states cannot be probed by NMR because these states contribute to the same lines of the ${ }^{13} \mathrm{C}$-NMR multiplet in a ${ }^{13} \mathrm{C}-\mathrm{D}_{3}$ group. Therefore, the populations of the E1 and E2 states can be lumped 
together: we do not consider the lumped populations $\mathcal{P}_{E 1}$ and $\mathcal{P}_{E 2}$ separately but instead introduce the total population of the E-manifold, $\mathcal{P}_{E}=\mathcal{P}_{E 1}+\mathcal{P}_{E 2}$. Hence, we will work in a reduced $3 \times 3$ manifold of spin states. In this situation, the relaxation rates are

$$
\begin{aligned}
\lambda_{1}=0, \lambda_{2} & =\frac{9 \tau_{C} \tau_{R}\left(2 \tau_{C}+\tau_{R}\right)}{2\left(\tau_{C}+\tau_{R}\right)\left(4 \tau_{C}+\tau_{R}\right)} \omega_{Q}^{2}, \\
\lambda_{3} & =\frac{3 \tau_{C} \tau_{R}\left(2 \tau_{C}+\tau_{R}\right)}{\left(\tau_{C}+\tau_{R}\right)\left(4 \tau_{C}+\tau_{R}\right)} \omega_{Q}^{2}
\end{aligned}
$$

and the eigenvectors corresponding to the lumped modes of the relaxation matrix are

$$
\mathcal{V}_{1}=\frac{1}{\sqrt{357}}\left(\begin{array}{c}
10 \\
1 \\
16
\end{array}\right), \mathcal{V}_{2}=\frac{1}{\sqrt{6}}\left(\begin{array}{c}
1 \\
1 \\
-2
\end{array}\right), \mathcal{V}_{3}=\frac{1}{\sqrt{42}}\left(\begin{array}{c}
5 \\
-1 \\
-4
\end{array}\right)
$$

The first mode again corresponds to the sum of all 27 populations, which must be a constant of motion. The coefficients reflect the dimensions of the sub-spaces and the fact that in thermal equilibrium, $\mathcal{P}_{A}=\frac{10}{27}, \mathcal{P}_{B}=\frac{1}{27}, \mathcal{P}_{E}=\frac{16}{27}$. The other two modes correspond to population imbalances that have long lifetimes due to their small auto-relaxation rates $\lambda_{2}$ and $\lambda_{3}$,

$$
\begin{aligned}
& \mathcal{M}_{2}=5 \mathcal{P}_{A}-\mathcal{P}_{B}-4 \mathcal{P}_{E}, \\
& \mathcal{M}_{3}=\mathcal{P}_{A}+\mathcal{P}_{B}-2 \mathcal{P}_{E} .
\end{aligned}
$$

These are the two observable long-lived modes in $\mathrm{CD}_{3}$-groups when the three-fold rotation is fast.

In principle, it is also possible to lump the states in one step, from $27 \times 27$ to $3 \times 3$ matrices, by directly lumping together all E states into a single state. However, for the sake of clarity, we broke the reduction down into two consecutive steps via a $4 \times 4$ matrix.

Detection of long-lived modes. To reveal manifestations of the long-lived modes $\mathcal{M}_{2}$ and $\mathcal{M}_{3}$ in the ${ }^{13} \mathrm{C}$-multiplet of a ${ }^{13} \mathrm{CD}_{3}$ group, we first express the intensities of the seven NMR lines of the septet, $L_{m}$ (where $m=1, \ldots, 7$ ), in terms of the populations $\mathcal{P}_{A}, \mathcal{P}_{B}, \mathcal{P}_{E}$. As explained above, we do not distinguish the populations of individual states within the three manifolds. This means that we neglect differences in intensities between NMR transitions that feature similar symmetry properties, for instance, the difference in intensity between transitions $L_{1}$ and $L_{7}$, which both correspond to the A manifold. Thus, we only consider linear combinations that depend on the total populations of the different symmetry manifolds,

$$
\left[\begin{array}{c}
\mathcal{L}_{a}=L_{1}+L_{7}=\frac{2}{10} \mathcal{P}_{A}, \mathcal{L}_{b}=L_{2}+L_{6}=\frac{2}{10} \mathcal{P}_{A}+\frac{4}{16} \mathcal{P}_{E} \\
2 \mathcal{L}_{b}=L_{3}+L_{5}=\frac{4}{10} \mathcal{P}_{A}+\frac{8}{16} \mathcal{P}_{E}, L_{4}=\mathcal{L}_{c}=\frac{2}{10} \mathcal{P}_{A}+\mathcal{P}_{B}+\frac{4}{16} \mathcal{P}_{E}
\end{array}\right.
$$

We readily see that two combinations, $\left(L_{2}+L_{6}\right)$ and $\left(L_{3}+L_{5}\right)$, are linearly dependent; hence, it is sufficient to consider their weighted sum $\left[2\left(L_{2}+L_{6}\right)+\left(L_{3}+L_{5}\right)\right] / 2$. Now we can express the averaged populations as a function of the line intensities,

$$
\mathcal{P}_{A}=5 \mathcal{L}_{a}, \mathcal{P}_{B}=\mathcal{L}_{c}-\mathcal{L}_{b}, \mathcal{P}_{E}=4\left(\mathcal{L}_{b}-\mathcal{L}_{a}\right) .
$$

Using these formulas, we can easily express the averaged populations of the long-lived modes, $\mathcal{M}_{2}$ and $\mathcal{M}_{3}$, via the NMR line intensities and extract them from experimental ${ }^{13} \mathrm{C}-\mathrm{NMR}$ multiplets. This allows us to monitor the time dependence of $\mathcal{M}_{2}$ and $\mathcal{M}_{3}$,

$$
\begin{aligned}
& \mathcal{M}_{2}=\frac{1}{2}\left(82 L_{1}-30 L_{2}-15 L_{3}-2 L_{4}-15 L_{5}-30 L_{6}+82 L_{7}\right), \\
& \mathcal{M}_{3}=13 L_{1}-9 L_{2}-\frac{9}{2} L_{3}+L_{4}-\frac{9}{2} L_{5}-9 L_{6}+13 L_{7} .
\end{aligned}
$$

To probe the existence and lifetime of the two longlived modes $\mathcal{M}_{2}$ and $\mathcal{M}_{3}$, we employed dissolution dynamic nuclear polarization (D-DNP), a method originally devised by Ardenkjaer-Larsen and co-workers in $2003^{3}$ and which has since developed into a powerful technique to investigate nonequilibrium quantum states that would remain unnoticed by means of conventional NMR spectroscopy. ${ }^{26}$ In brief, a solid sample of deuterated ethanol- $\mathrm{d}_{6}$ or DMSO- $\mathrm{d}_{6}$, both containing a ${ }^{13} \mathrm{CD}_{3}$ moiety, supplemented with $50 \mathrm{mM}$ of the nitroxide TEMPOL and $50 \%$ v/v glycerol- $\mathrm{d}_{8}$ as cryo-protectant, was irradiated for $3 \mathrm{~h}$ by microwaves with a frequency near 188.2 GHz (modulated over a range of $100 \mathrm{MHz}$ with a modulation frequency of $2 \mathrm{kHz}$, and a power of ca. $35 \mathrm{~mW}$ at the locus of the sample) in a prototype dynamic nuclear polarization (DNP) apparatus operating at $1.2 \mathrm{~K}$ in a magnetic field of $6.7 \mathrm{~T}$. In this manner, the $\mathrm{D}$ as well as ${ }^{13} \mathrm{C}$ nuclei present in the ${ }^{13} \mathrm{CD}_{3}$ groups are simultaneously hyperpolarized leading to an overpopulation of the lowest or highest energy state, depending on the microwave frequency. The symmetry of the predominantly populated spin state depends on a multitude of factors including the microwave frequency and the methyl group tunneling probability; ${ }^{13}$ a detailed discussion of these factors is beyond the scope of this manuscript. For the case at hand, it suffices to note that DNP at low temperatures will overpopulate one of the symmetry manifolds.

After hyperpolarization, the sample it is rapidly dissolved by a burst of $5 \mathrm{~mL} \mathrm{D}_{2} \mathrm{O}$ pressurized to $1.05 \mathrm{MPa}$, squirted onto the sample. The dissolved sample is transported within $4 \mathrm{~s}$ to a 9.4 T NMR spectrometer where NMR spectra are taken in a time-resolved manner every second by applying $30^{\circ}$ detection pulses on the ${ }^{13} \mathrm{C}$ nuclei of the ${ }^{13} \mathrm{CD}_{3}$ groups. The septets are visible for about $2 \mathrm{~min}$, at which time the carbon polarization becomes too small to be detected in a single scan.

It is important to note that the longitudinal deuterium relaxation, as characterized by $T_{1}(\mathrm{D})$, is rather fast, typically on the order of a second. We can thus safely assume that the 
a)

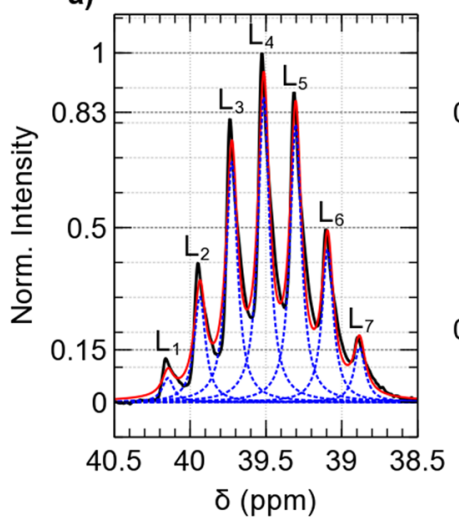

b)

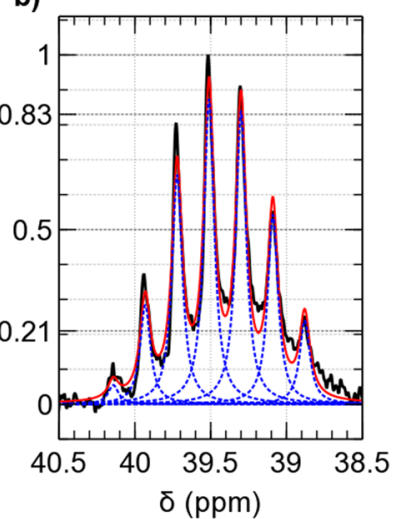

c)

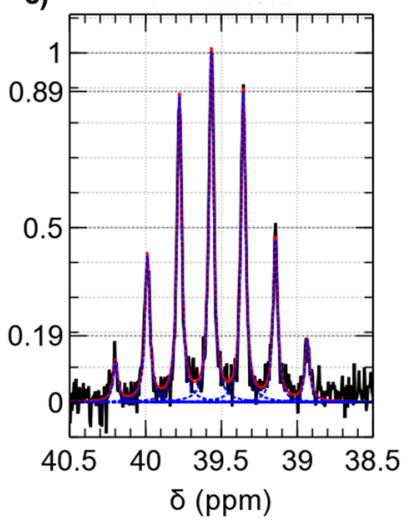

FIG. 2. (a) DNP-hyperpolarized ${ }^{13} \mathrm{C}$ multiplet of the ${ }^{13} \mathrm{CD}_{3}$ groups in DMSO- $\mathrm{d}_{6} 10 \mathrm{~s}$ after injection into a $9.4 \mathrm{~T}$ NMR spectrometer. (b) DNP-hyperpolarized ${ }^{13} \mathrm{C}$ multiplet of the ${ }^{13} \mathrm{CD}_{3}$ group in ethanol- $\mathrm{d}_{6} 30 \mathrm{~s}$ after injection into a $9.4 \mathrm{~T}$ NMR spectrometer. (c) Multiplet of the ${ }^{13} \mathrm{CD}_{3}$ group of DMSO-d ${ }_{6}$ in thermal equilibrium with an intensity distribution 1:3:5:7:5:3:1. Note that the lines are much narrower in thermal equilibrium than in the hyperpolarized case. This may result from convection and small helium gas bubbles that perturb the homogeneity. The intensity ratios between different lines are not perturbed through this.

Zeeman distribution of the $\mathrm{D}_{3}$ system is in thermal equilibrium after the dissolution and transfer which take about $4 \mathrm{~s}$. In other words, within each of the symmetry manifolds, A, B, and $\mathrm{E}$, the distribution of populations is in thermal equilibrium during the detection period. All deviations of the septets from their thermal equilibrium intensities of $1: 3: 5: 7: 5: 3: 1$ at times longer than $T_{1}$ (D) can therefore be attributed to the presence of long-lived SSI.

Typical spectra resulting from hyperpolarization and dissolution are displayed in Fig. 3 for the ${ }^{13} \mathrm{CD}_{3}$ groups of DMSO-d ${ }_{6}$, once $10 \mathrm{~s}$ after injection (Fig. 2(a)), after $30 \mathrm{~s}$ (Fig. 2(b)), and in thermal equilibrium (Fig. 2(c), averaged over 3584 scans). To determine the intensities of the 7 lines, each one was individually fitted to a Lorentzian (dashed blue lines in Fig. 2). The intensities (integrals) $L_{i}$ of the 7 lines (the numbering is indicated in Fig. 2(a)) were normalized to the central line $L_{4}$. Details on the determination of the integral values are given in Sec. IV. In brief, the spectra were fitted to 7 independent Lorentzians of similar linewidth and split by coupling constants $J_{\mathrm{CD}}(\mathrm{EtOD})=19.1 \mathrm{~Hz}$ and $J_{\mathrm{CD}}(\mathrm{DMSO})=21.2 \mathrm{~Hz}$. The method was adapted from a procedure developed by Miclet et al. ${ }^{19}$ Here we used a Nelder algorithm for fitting. All peaks were fitted with a similar linewidth and the different lines are separated by a single J-coupling. For details, see Sec. IV.

Deviations of the intensities of the seven lines from their equilibrium intensity distribution 1:3:6:7:6:3:1 hint toward non-equilibrium deuterium polarizations. For example, the relative intensities $L_{1}$ and $L_{7}$ are smaller after hyperpolarization than at thermal equilibrium. (As we show below, this is because the E-states are overpopulated.) The very high signal intensities in D-DNP experiments allows us to access such subtle differences.

The carbon nucleus of the ${ }^{13} \mathrm{CD}_{3}$ group is also hyperpolarized after dissolution (Fig. 2(a)). Its enhancement factor was ca. 22000 corresponding to a ${ }^{13} \mathrm{C}$ polarization of ca. $18 \%$ directly after dissolution and transfer. Similar observations were made for ethanol- $\mathrm{d}_{6}$. a)

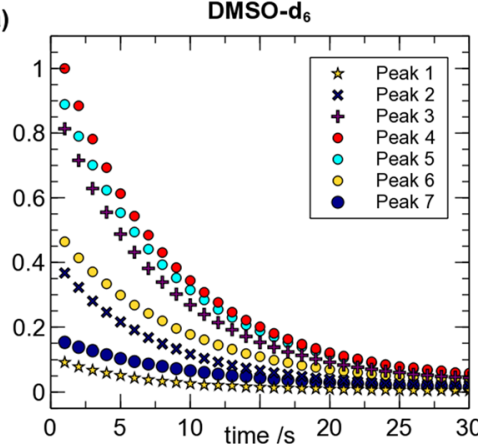

c)

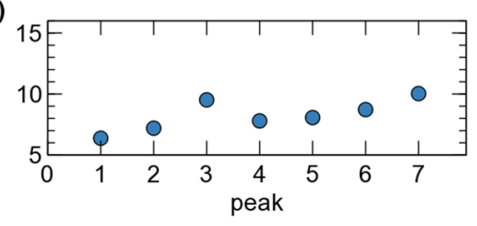

b)

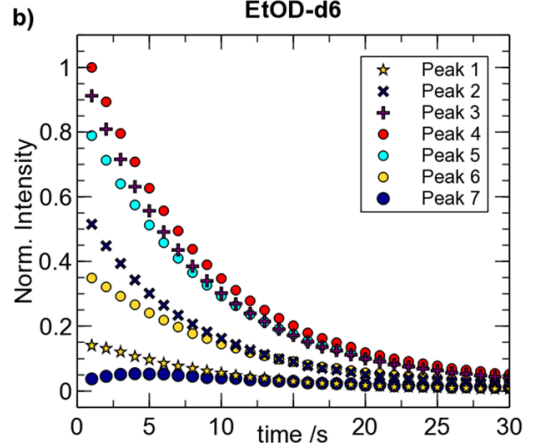

d)

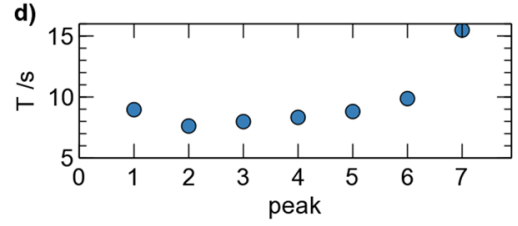

FIG. 3. (a) Decays of the seven individual lines of the ${ }^{13} \mathrm{C}$ septet of DMSO-d 6 (numbered as in Fig. 2) after hyperpolarization, dissolution, and transfer. (b) Similar data for ethanol- $\mathrm{d}_{6}$. (c) Characteristic relaxation times of the seven lines of the ${ }^{13} \mathrm{C}$ septet in DMSO- $\mathrm{d}_{6}$. (d) Characteristic relaxation times of the seven transitions in ethanol- $\mathrm{d}_{6}$. The relaxation times shown in (c) and (d) have been extracted assuming mono-exponential decays. For peak 7, only the decreasing part of the time trace was considered. 
After hyperpolarization, dissolution, and transfer, the carbon polarization decays toward thermal equilibrium, a process that is dominated by the longitudinal auto-relaxation rate $1 / T_{1}\left({ }^{13} \mathrm{C}\right)$. This decay is documented in Fig. 3 for both molecules. Each line of the carbon septet is monitored individually in Fig. 3(a) for DMSO- $\mathrm{d}_{6}$. The time traces were fitted to mono-exponential decay functions. The corresponding relaxation times are shown in Fig. 3(b), likewise for ethanol- $\mathrm{d}_{6}$ in Figs. 3(c) and 3(d). The relaxation times of the different lines of the carbon septet span a range between 6 and 12 s, i.e., by a factor up to two. This difference cannot be accounted by the here presented theory as it involves the carbon nucleus of the $\mathrm{CD}_{3}$ group, but is a consequence of the presence of an SSI as has been shown in our earlier work. $^{15}$

To analyze the SSIs spanning the ${ }^{13} \mathrm{CD}_{3}$ groups, we calculated the values $\mathcal{P}_{A}, \mathcal{P}_{B}$, and $\mathcal{P}_{E}$ according to Eq. (19) as well as the two long-lived modes $\mathcal{M}_{2}$ and $\mathcal{M}_{3}$ according to Eqs. (17) and (19) for both DMSO- $\mathrm{d}_{6}$ and ethanol- $\mathrm{d}_{6}$. The results are shown in Figs. 4 and 5. To remove biases due to carbon relaxation from the analysis, we normalized each line to the total intensity summed over the seven lines before applying Eqs. (17)-(19) to the integrated intensities of the seven individual lines. Figures 5(a) and 5(b) show the time dependence after hyperpolarization, dissolution, and transfer to the detection spectrometer of three different populations $\mathcal{P}_{A}, \mathcal{P}_{B}$, and $\mathcal{P}_{E}$. Evidently, the time trace for $\mathcal{P}_{B}$ is flat, indicating that DNP most likely does not affect the population of the $\mathrm{B}$ manifold. On the contrary, the populations $\mathcal{P}_{A}$ and $\mathcal{P}_{E}$ show a strong time dependence, which is increasing for $\mathcal{P}_{A}$ and decreasing for $\mathcal{P}_{E}$. This behavior was observed for both molecules. These observations can be interpreted as an imbalance that starts with an overpopulation of the E manifold and a depleted population
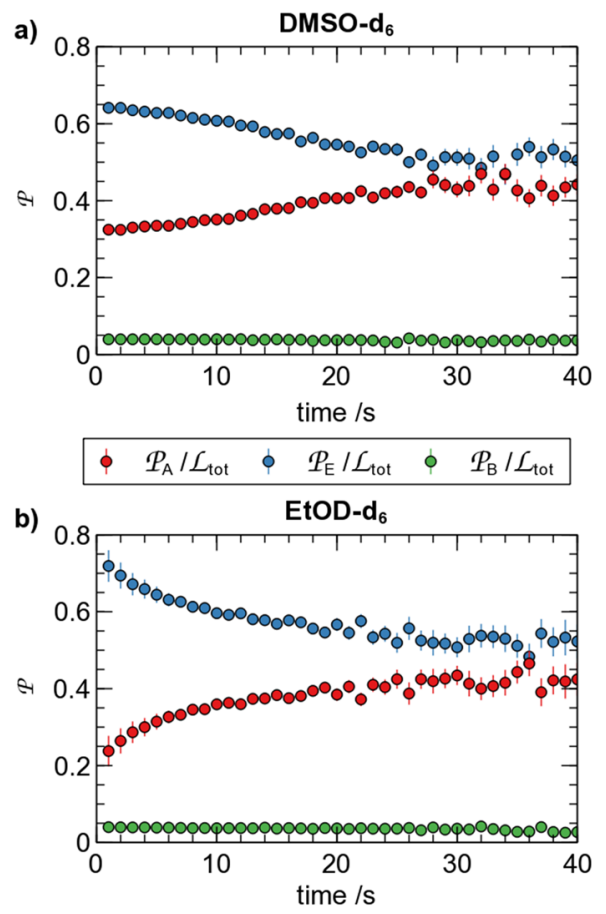

FIG. 4. (a) Time dependence of $\mathcal{P}_{A}, \mathcal{P}_{B}$, and $\mathcal{P}_{E}$ in DMSO-d 6 after hyperpolarization and dissolution. (b) Similar data for ethanol- $\mathrm{d}_{6}$.
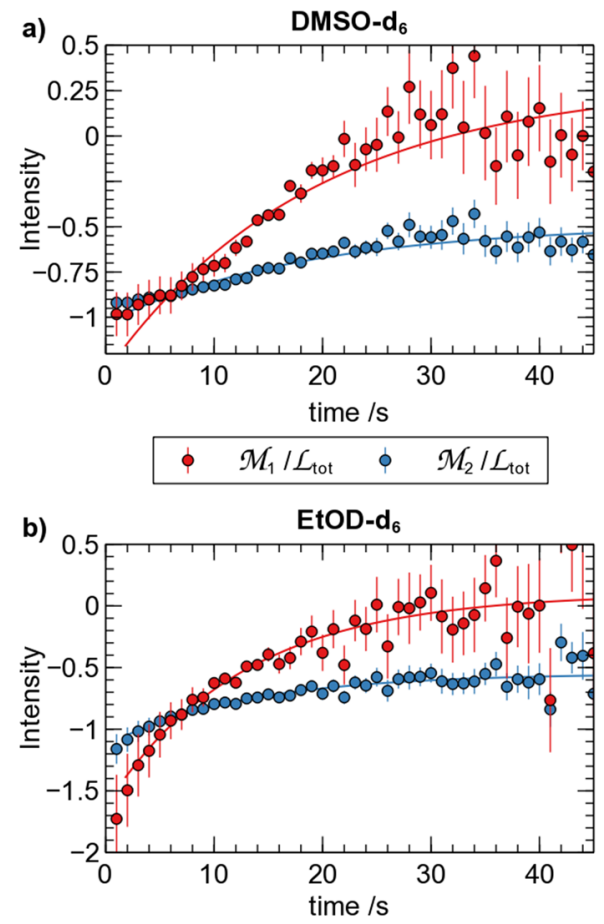

FIG. 5. (a) Decays of the two long lived modes $\mathcal{M}_{1}$ and $\mathcal{M}_{2}$ after hyperpolarization, dissolution, and transfer for DMSO-d $\mathrm{d}_{6}$. (b) Similar decays for ethanol- $\mathrm{d}_{6}$. All data are normalized by dividing by the total intensity of the carbon septets to cancel the effects of $T_{1}\left({ }^{13} \mathrm{C}\right)$ from the analysis.

of the A manifold. These imbalances decay with time toward their thermal equilibrium values. However, the decay does not appear to be mono-exponential, likely due to cross-relaxation phenomena.

In Figs. 5(a) and 5(b), we calculated the time dependence of the two long-lived modes $\mathcal{M}_{2}$ and $\mathcal{M}_{3}$ from the populations $\mathcal{P}_{A}, \mathcal{P}_{B}$, and $\mathcal{P}_{E}$. Both modes decay with time, which can be attributed to the imbalance identified above which enters the definition of Eq. (17). We find a characteristic relaxation time of $19.0 \pm 2.2 \mathrm{~s}$ for $\mathcal{M}_{2}$ and $18.5 \pm 2.1 \mathrm{~s}$ for $\mathcal{M}_{3}$ for DMSO-d $\mathrm{d}_{6}$. The $T_{1}\left({ }^{2} \mathrm{H}\right)$ auto-relaxation time in DMSO- $\mathrm{d}_{6}$ has been determined to be $0.7 \mathrm{~s}$ for the ${ }^{12} \mathrm{CD}_{3}$ groups of DMSO- $\mathrm{d}_{6}$. This yields a prolongation of the relaxation time of deuterium by the factor of $19.0 / 0.7=27.1$. This finding is in good agreement with earlier results. ${ }^{15}$ For ethanol- $\mathrm{d}_{6}$, we find a decay time of $12.6 \pm 2.4 \mathrm{~s}$ for $\mathcal{M}_{2}$ and $12.7 \pm 2.4 \mathrm{~s}$ for $\mathcal{M}_{3}$, while $T_{1}\left({ }^{2} \mathrm{H}\right)=2.7 \mathrm{~s}$, which is astonishingly long for deuterons and points toward fast rotation of the ${ }^{13} \mathrm{CD}_{3}$ group and results in a prolongation of $12.6 / 2.7=4.7$. Note that in Fig. $5, \mathcal{M}_{2}$ and $\mathcal{M}_{3}$ have been normalized by the integral of the septets. This assures the absence of bias due to ${ }^{13} \mathrm{C}$ auto-relaxation but causes the "noisy" appearance of the time dependence at longer times.

Our simplified approach cannot account for the observation of significant differences between the intensities $L_{1}$ and $L_{7}$, as well as between the pairs $L_{2}, L_{6}$, and $L_{3}, L_{5}$, i.e., between pairs of lines that appear symmetrically with respect to the center of the septet. Such asymmetries are a consequence of the coupling of deuterium SSIs to the detected carbon-13 nucleus, or of SSIs that cross-relax into multispin order terms, which produce antiphase carbon magnetization with respect to the 
attached deuterons upon detection (see Kurzbach et $a l .{ }^{16}$ ). However, these deviations are not considered here since the combinations underlying the modes $\mathcal{M}_{1}$ and $\mathcal{M}_{2}$ sum over pairs of symmetrically disposed and therefore ignore such asymmetries.

\section{CONCLUSIONS}

The analysis of symmetry properties in spin systems such as the $\mathrm{CD}_{3}$ groups presented here is challenging because of the dimensions of the Liouville space; hence, the corresponding relaxation matrices become quite large. The proposed method here for the reduction of the dimensionality of relaxation matrices helps to identify important lumped modes and relaxation properties. Indeed, with our method, we can identify longlived spin state imbalances in $\mathrm{CD}_{3}$ groups and analytically calculate their lifetimes avoiding diagonalization of the original $27 \times 27$ relaxation matrix. Using this method, we find that the quadrupolar relaxation of long-lived modes in $\mathrm{CD}_{3}$ groups is independent of the overall tumbling of the molecule containing the $\mathrm{CD}_{3}$ groups, but depends-according to our calculations - only on the rotational correlation time of the methyl groups describing methyl group jumps. This method might help to predict long-lived states in even more complex systems and extend applications of long-lived spin states to NMR spectroscopy and imaging. In this work, we confirm that long-lived modes can be generated and analyzed for spin-1 nuclei, such as ${ }^{2} \mathrm{H}$. We anticipate that such longlived states can be utilized for probing dynamics of various molecules and for extending the D-DNP methodology to ${ }^{2} \mathrm{H}$ nuclei.

\section{EXPERIMENT}

Samples were prepared by mixing $50 \% \mathrm{v} / \mathrm{v}$ of glycerol- $\mathrm{d}_{8}$ with either of two different solutions of $50 \mathrm{mM}$ TEMPOL in $80 \%$ DMSO-d6/10\% $\mathrm{H}_{2} \mathrm{O}$ or in $80 \%$ ethanol-d6/10\% $\mathrm{H}_{2} \mathrm{O}$. Using a Bruker BioSpin prototype D-DNP apparatus, $50 \mu \mathrm{L}$ of each sample were hyperpolarized at $1.2 \mathrm{~K}$ and $6.7 \mathrm{~T}$ for ca. $2 \mathrm{~h}$ by direct microwave irradiation near $188.38 \mathrm{GHz}$. The microwave carrier frequency was modulated with a saw-tooth wave over a range of $100 \mathrm{MHz}$ with a modulation frequency of $2 \mathrm{kHz}$.

The samples were dissolved in $5 \mathrm{~mL} \mathrm{D}_{2} \mathrm{O}$ at $180{ }^{\circ} \mathrm{C}$ under 1.05 MPa pressure and propelled by helium gas within $3 \mathrm{~s}$ via a magnetic tunnel that maintains a minimum field near 0.9 $\mathrm{T}$ to a $9.4 \mathrm{~T}$ detection NMR spectrometer equipped with a $10 \mathrm{~mm}$ broadband " $\beta$-barium borate $(\mathrm{BBO})$ " probe for ${ }^{13} \mathrm{C}$ detection. The temperature was adjusted to $298 \mathrm{~K}$. Radiofrequency pulses with $30^{\circ}$ nutation angles were applied every $1 \mathrm{~s}$ for detection. The data were processed, zero-filled, and apodized using NMRPipe, ${ }^{11}$ and analyzed with home-written scripts using the SciLab software package. ${ }^{14}$

The envelopes $I_{\text {exp }}$ of the 7 experimental lines were fitted by minimizing $I_{\text {exp }}-I_{f i t_{2}}$ by a Nelder algorithm to reproduce the shape of the spectrum. A common linewidth was fitted to all seven equidistant peaks, while the individual intensities were fitted independently. Errors were determined via Gaussian propagation of the differences between the experimental and fitted intensities.

\section{ACKNOWLEDGMENTS}

The authors thank Bruker BioSpin for the prototype D-DNP equipment. This work was supported by the French CNRS and the European Research Council (contract "Dilute para-water"). K.L.I. acknowledges ENS for a guest professorship and the Russian Science Foundation (Grant No. 14-13-01053).

\section{APPENDIX: SYMMETRY-ADAPTED SPIN STATES}

In this Appendix, we briefly discuss symmetry-adapted states. ${ }^{6}$ The total spin $F$ of the three $S=1$ spins can be equal to 3,2 , 1 , or 0 . The $F=3$ states belong to the first $A$-manifold of seven states, which remain unchanged upon cyclic permutations of the 3 spins. For instance,

$$
|3,3 A\rangle=|111\rangle,|3,2 A\rangle=\frac{1}{\sqrt{3}}(|110\rangle+|101\rangle+|011\rangle),
$$

etc. In the notation of symmetry-adapted states, $\mid F, M$ irrep $\rangle$, we define the total spin $F$, its $z$-projection $M$, and the irreducible representation, irrep; in the product states $\left|m_{1} m_{2} m_{3}\right\rangle$, we specify the $z$-projections of all three D-spins. The states with $F=2$ are the E1 and E2 states, which are invariant upon the pairwise exchange of spins. For instance, the state

$$
|2,2 E 1\rangle=\frac{1}{\sqrt{6}}(2|110\rangle-|101\rangle-|011\rangle)
$$

is invariant upon the exchange of spins 1 and 2, whereas the state

$$
|2,2 E 2\rangle=\frac{1}{\sqrt{2}}(|101\rangle-|011\rangle)
$$

is anti-symmetric upon the exchange of spins 1 and 2. The definitions of the E1 and E2 states in Eqs. (A2) and (A3) are not unique since one can choose arbitrary linear combinations of these states. This is not essential in this work since the E1 and E2 states can be "lumped together" because the corresponding ${ }^{13} \mathrm{C}$ transitions overlap. The reader is referred to Refs. 5-7 for a more detailed treatment. The $F=1$ states are the $A$, $E 1$, and $E 2$ states and have similar symmetry properties as the $A, E 1$, and $E 2$ states discussed above. For instance, the state

$$
\left|1,1 A=\frac{1}{\sqrt{15}}(2|11-1\rangle+2|1-11\rangle+2|-111\rangle-|100\rangle-|010\rangle-|001\rangle)\right.
$$

is invariant to cyclic permutations of the three spins, but the $E 1$ and $E 2$ states remain the same or change the sign when spins 1 and 2 are exchanged, 


$$
\begin{aligned}
& |1,1 E 1\rangle=\frac{1}{\sqrt{12}}(2|11-1\rangle-|1-11\rangle-|-111\rangle+2|001\rangle-|100\rangle-|010\rangle) \\
& |1,1 E 2\rangle=\frac{1}{2}(|1-11\rangle-|-111\rangle+|010\rangle-|100\rangle)
\end{aligned}
$$

Finally, there is a single $F=0$ state, which is the B-state,

$$
|0,0 B\rangle=\frac{1}{\sqrt{6}}(|10-1\rangle+|0-11\rangle+|-110\rangle-|01-1\rangle-|-101\rangle-|1-10\rangle) .
$$

This state is invariant to cyclic permutations of the three D-spins.

${ }^{1}$ Ahuja, P., Sarkar, R., Vasos, P. R., and Bodenhausen, G., J. Chem. Phys. 127, 134112-1 (2007).

${ }^{2}$ Ahuja, P., Sarkar, R., Vasos, P. R., and Bodenhausen, G., J. Am. Chem. Soc. 131, 7498 (2009).

${ }^{3}$ Ardenkjaer-Larsen, J. H., Fridlund, B., Gram, A., Hansson, G., Hansson, L., Lerche, M. H., Servin, R., Thaning, M., and Golman, K., Proc. Natl. Acad. Sci. U. S. A. 100, 10158 (2003).

${ }^{4}$ Bengs, C. and Levitt, M. H., Magn. Reson. Chem. 56, 374 (2017).

${ }^{5}$ Bernatowicz, P. and Szymanski, S., Mol. Phys. 101, 353 (2003).

${ }^{6}$ Bernatowicz, P., Kowalewski, J., Kruk, D., and Werbelow, L. G., J. Phys. Chem. A 108, 9018 (2004).

${ }^{7}$ Bernatowicz, P., Kowalewski, J., Kruk, D., and Werbelow, L. G., J. Phys. Chem. A 108, 9018 (2004).

${ }^{8}$ Bornet, A., Ahuja, P., Sarkar, R., Fernandes, L., Hadji, S., Lee, S. Y., Haririnia, A., Fushman, D., Bodenhausen, G., and Vasos, P. R., ChemPhysChem 12, 2729 (2011).

${ }^{9}$ Carravetta, M. and Levitt, M. H., J. Am. Chem. Soc. 126, 6228 (2004).

${ }^{10}$ Carravetta, M., Johannessen, O. G., and Levitt, M. H., Phys. Rev. Lett. 92, 153003 (2004).

${ }^{11}$ Delaglio, F., Grzesiek, S., Vuister, G. W., Zhu, G., Pfeifer, J., and Bax, A., J. Biomol. NMR 6, 277 (1995).

${ }^{12}$ Dumez, J. N., Hakansson, P., Mamone, S., Meier, B., Stevanato, G., Hill-Cousins, J. T., Roy, S. S., Brown, R. C. D., Pileio, G., and Levitt, M. H., J. Chem. Phys. 142, 044506 (2015).

${ }^{13}$ Dumez, J. N., Vuichoud, B., Mammoli, D., Bornet, A., Pinon, A. C., Stevanato, G., Meier, B., Bodenhausen, G., Jannin, S., and Levitt, M. H., J. Phys. Chem. Lett. 8, 3549 (2017).
${ }^{14}$ Enterprises, S. S. Scilab: Le logiciel open source gratuit de calcul numérique, 2012.

${ }^{15}$ Jhajharia, A., Weber, E. M. M., Kempf, J., Abergel, D., Bodenhausen, G., and Kurzbach, D., J. Chem. Phys. 146, 041101 (2017).

${ }^{16}$ Kurzbach, D., Weber, E. M. M., Jhajharia, A., Cousin, S. F., Sadet, A., Marhabaie, S., Canet, E., Birlirakis, N., Milani, J., Jannin, S., Eshchenko, D., Hassan, A., Melzi, R., Luetolf, S., Sacher, M., Rossire, M., Kempf, J., Lohman, J. A. B., Weller, M., Bodenhausen, G., and Abergel, D., J. Chem. Phys. 145, 194203 (2016).

${ }^{17}$ Levitt, M. H., "Singlet nuclear magnetic resonance," in Annual Review of Physical Chemistry, edited by Johnson, M. A. and Martinez, T. J. (Annual Reviews, Palo Alto, 2012), Vol. 63, pp. 89.

${ }^{18}$ Meier, B., Dumez, J. N., Stevanato, G., Hill-Cousins, J. T., Roy, S. S., Hakansson, P., Mamone, S., Brown, R. C. D., Pileio, G., and Levitt, M. H., J. Am. Chem. Soc. 135, 18746 (2013).

${ }^{19}$ Miclet, E., Abergel, D., Bornet, A., Milani, J., Jannin, S., and Bodenhausen, G., J. Phys. Chem. Lett. 5, 3290 (2014).

${ }^{20}$ Pileio, G., Prog. Nucl. Magn. Reson. Spectrosc. 56, 217 (2010).

${ }^{21}$ Pileio, G., Prog. Nucl. Magn. Reson. Spectrosc. 98-99, 1 (2017).

${ }^{22}$ Pileio, G., Dumez, J. N., Pop, I. A., Hill-Cousins, J. T., and Brown, D. R. C., J. Magn. Reson. 252, 130 (2015).

${ }^{23}$ Poupko, R., Vold, R. L., and Vold, R. R., J. Magn. Reson. 34, 67 (1979).

${ }^{24}$ Sarkar, R., Vasos, P. R., and Bodenhausen, G., J. Am. Chem. Soc. 129, 328 (2007).

${ }^{25}$ Sarkar, R., Ahuja, P., Vasos, P. R., and Bodenhausen, G., ChemPhysChem 9, 2414 (2008).

${ }^{26}$ Tayler, M. C., Marco-Rius, I., Kettunen, M. I., Brindle, K. M., Levitt, M. H., and Pileio, G., J. Am. Chem. Soc. 134, 7668 (2012). 